\title{
Roles for Mitotic History in the Generation and Degeneration of Hippocampal Neuroarchitecture
}

\author{
Mark P. Mattson, Peter B. Guthrie, Barbara C. Hayes, and S. B. Kater \\ Program in Neuronal Growth \& Development, and Department of Anatomy and Neurobiology, Colorado State University, \\ Fort Collins, Colorado 80523
}

\begin{abstract}
The mechanisms regulating the highly ordered neuroarchitecture of the mammalian brain are largely unknown. The present study took advantage of hippocampal pyramidallike neurons that arose from a common progenitor cell in cell culture (sister neurons) to ascertain the contribution of intrinsic factors to both the generation and degeneration of neuroarchitecture. Sister neurons were similar in overall cell form and dendritic numbers and lengths. Control non-sister neurons that grew in contact did not generate similar morphologies, indicating that the similarity of sister cells did not result from influences of the local microenvironment or cell interactions. These results suggest that intrinsic factors related to mitotic history play a role in the generation of neuroarchitecture. Since particular groups of hippocampal neurons are sensitive to glutamate neurotoxicity in situ and are vulnerable in neurodegenerative disorders, it was of interest to test glutamate sensitivity in the neuronal population and in mitotic sister neurons. A subpopulation of pyramidal neurons was sensitive to glutamate neurotoxicity. A striking finding was that sister neurons were invariably either both sensitive or both resistant to glutamate, while non-sister neurons often showed different responses to glutamate. Pharmacological studies indicated that glutamate neurotoxicity was mediated by kainate/quisqualate type receptors by a mechanism involving calcium influx through membrane channels. Fura-2 measurements of intracellular calcium revealed that sister neurons had similar rest levels of calcium and, strikingly, glutamate caused a dramatic increase in intracellular calcium levels only in neurons which subsequently degenerated. Apparently, intrinsic differences in sensitivity to glutamate lie at a point prior to calcium entry, probably at the level of glutamate receptors. Taken together, these results indicate that the mitotic history of a neuron can determine its presence and potential for connectivity as well as its susceptibility to neurodegeneration.
\end{abstract}

The physical form of a neuron is a fundamental determinant of its information coding capabilities within the nervous system.

\footnotetext{
Received May 24, 1988; revised Aug. 31, 1988; accepted Oct. 12, 1988.

We thank Dr. G. Banker for help in establishing the culture procedures; P. Dou and D. Giddings for technical assistance; and Drs. K. Beam, M. V. L. Bennett, D. Gould, S. Kinnamon, and S. Roper for critical comments on the manuscript. Supported by NIH grants NS 08054 (M.P.M.); NS 24683, NS24561, and NS1 5350 (S.B.K.); and a fellowship to M.P.M. from the John Douglas French Foundation for Alzheimer's Disease.

Correspondence should be addressed to Dr. Mark P. Mattson at his present address: Sanders-Brown Center on Aging and Department of Anatomy \& Neurobiology, 211 Sanders-Brown Building, University of Kentucky Medical Center, Lexington, KY 40536-0230.

Copyright (C) 1989 Society for Neuroscience $0270-6474 / 89 / 041223-10 \$ 02.00 / 0$
}

The cellular mechanisms regulating the establishment, adult plasticity, and degeneration of neuroarchitecture are largely unknown. Considerable evidence indicates that environmental factors such as growth factors (Levi-Montalcini and Angeletti, 1968; Greene and Shooter, 1980), neurotransmitters (Haydon et al., 1984; Lankford et al., 1987; Lipton et al., 1988; Mattson, 1988; Mattson et al., 1988a), and growth substrates (Letourneau, 1985; Mattson and Kater, 1988a) are important regulators of neuronal outgrowth and survival. Recent studies of cultured hippocampal neurons demonstrated that glutamate, a major excitatory neurotransmitter in the mammalian brain (Fonnum, 1984), can selectively affect dendritic outgrowth and synaptogenesis in pyramidal neurons (Mattson et al., 1988a, b). The mechanism by which glutamate alters dendritic outgrowth involves calcium influx through plasma membrane channels (Mattson et al., 1988a, d). These results suggested that glutamate may play a role in the development of the very circuits in which it codes information in learning and memory processes (Lynch, 1986).

In addition to possible roles in development, glutamate may also be involved in neurodegeneration associated with aging and disease. Evidence gathered both in vivo and in vitro indicates that glutamate may be involved in the selective loss of hippocampal neurons in conditions such as Alzheimer's disease, epilepsy, and stroke (Coyle et al., 1981; Rothman, 1984; Schwarcz et al., 1984; Meldrum, 1985; Maragos et al., 1987; Mattson, 1988). One intriguing question related to neurodegeneration in the hippocampus is why particular groups of pyramidal neurons are vulnerable. For example, in Alzheimer's disease neuronal loss is prominent in region CA1 (VanHoesen et al., 1986), while in epilepsy the CA 3 and CA 1 pyramidal neurons are particularly vulnerable (Scheibel et al., 1974; Sloviter, 1983; Paul and Scheibel, 1986). The cellular mechanism of glutamate neurotoxicity apparently involves a large rise in intracellular calcium levels resulting from sustained membrane depolarization (Rothman, 1984; Choi, 1987; Mattson and Kater, 1989; Mattson et al., $1988 \mathrm{a}, \mathrm{d})$. The basis for the selective vulnerability of different neurons is not clear but may result from differences in the $\mathrm{mi}$ croenvironment (e.g., position within the neuronal circuitry) and/or intrinsic neuronal properties (e.g., expression of glutamate receptors).

In comparison to our knowledge of environmental factors that affect ncuronal cytoarchitccturc, the relative contributions of intrinsic neuronal properties have gone largely unexplored (but see Kater and Mattson, 1988; Lasek and Black, 1988). Invertebrates such as nematodes, leeches, and insects have proven useful for cell lineage studies of neuronal development since, in these organisms identified neurons arise from nearly invariant patterns of cell division (Goodman, 1982; Stent et al., 1982; 
Sulston et al., 1983). Roles for cell lineage in determining neuroarchitecture in vertebrate nervous systems are unclear, although a few studies have suggested the importance of cell lineage (Herrup, 1987). In addition, studies of neuroblastoma cells (Solomon, 1981) indicated that cells that are closely related through mitosis also tend to be related morphologically. Appropriate model systems and new technical approaches such as retroviral markers (Luskin et al., 1987) are clearly important to furthering our understanding of roles for cell lineage in the generation and degeneration of mammalian neuroarchitecture. In the present study we employed an in vitro model of hippocampal development in which the mitosis and subsequent neuronal differentiation of embryonic rat hippocampal cells can be reliably studied in cell culture. We report that sister pyramidal neurons that arise from a common progenitor are morphologically related and invariably show identical sensitivities to glutamate neurotoxicity. Selective vulnerability appears to result from differences in the expression of glutamate receptors linked to calcium influx.

Portions of this work were presented at the First International Conference on Alzheimer's Disease and Related Disorders (Las Vegas, 1988).

\section{Materials and Methods}

Hippocampal cell cultures. Cultures were established from hippocampi of embryonic day 17 (E17) rats. (Timed pregnant rats were obtained from Harlan Sprague-Dawley, Indianapolis, IN.) The culture methods are described elsewhere (Mattson et al., 1988a; Mattson and Kater, 1988b). Pyramidal neurons were identified by morphological criteria (Banker and Cowan, 1977; Dotti et al., 1988; Mattson et al., 1988a). Neurons were visualized and photographed with a phase-contrast Nikon Diaphot inverted microscope. At stage E17, approximately $10-20 \%$ of the presumptive pyramidal cells have not yet undergone their last mitosis, and cell division can be observed in culture (Banker and Cowan, 1977; Mattson et al., 1988a). Within 4-6 hr of plating, cytokinesis can be observed in a small percentage (approximately $5 \%$ ) of the cells (e.g., Fig. $1 \mathrm{~A}$ ). By $20-30 \mathrm{hr}$ postplating approximately $10-20 \%$ of the cells have undergone mitosis and are differentiated as pyramidal-like neurons (e.g., Fig. 1 $A^{\prime \prime}$ ). Mitotic pairs of neurons generated from the same precursor cell were identified as such by following their developmental progress in culture (Fig. 1). Neurons were relocated by virtue of their positions with respect to markings etched on the outer surface of the culture dishes; neurons retained their positions in the culture dish throughout the course of the experiments. Only pairs of neurons arising from a division between 4 and $20 \mathrm{hr}$ in culture were used for analyses. Under the low density culture conditions employed, cell dissociation was virtually complete and less than $1 \%$ of the cells were in physical contact after attaching to the polylysine culture substrate. These nonsister cell pairs that grew in contact with each other, though rare, served as useful controls for examining the possible influence of microenvironment and cell interactions on the generation of morphology and sensitivity to glutamate (see below).

Assessments of neuronal morphology and cell survival. Tracings of ncurons made from projected photographic negatives of pictures taken on culture day 3 were used to analyze neuronal morphology. Only neuron pairs, be they sisters or non-sisters, that had initiated outgrowth between 4 and $20 \mathrm{hr}$ of plating were evaluated. Overall similarities in pyramidal cell form were assessed both qualitatively and quantitatively. In one experiment naive subjects were given sets of 20 cards, each card containing a tracing of one of a pair of sister neurons. The subjects were asked to arrange the cards so as to pair neurons with similar morphologies; the number of correct pairings of sister neurons was recorded. Random pairing trials in which cards were paired blindly were used to determine the chance level of pairing. For quantitative analyses, the number of primary dendrites emanating from the soma were counted and total dendritic length for each neuron was measured using the calibration provided by a micrometer slide. Cell viability was determined on culture day 5 ; cultures were exposed to experimental treatments and cell viability was assessed $4 \mathrm{hr}$ later. In general, neurons susceptible to glutamate toxicity were either absent or had swollen, rounded somas and fragmented neurites. Glutamate-resistant neurons showed to somal swelling or neurite fragmentation and retained active growth cones (e.g., Fig. 3); these resistant neurons survived at least $20 \mathrm{hr}$ in the presence of glutamate. The results of an initial experiment showed that cell viability according to these morphological criteria agreed within $5 \%$ of counts of trypan blue-excluding neurons $(0.4 \%$ solution in $0.15 \mathrm{~m}$ saline). Glutamate, kainate, quisqualate, $\mathrm{N}$-methyl-D-aspartic acid (NMDA), gamma-D-glutamylglycine (DGG), D,L-2-amino-5-phosphonovaleric acid (APV), and GABA (all from Sigma) were prepared as stocks in culture medium and were added to cultures in 22 or 220 $\mu$ l volumes. A23187 (Sigma) and diazepam (Elkins-Sinn, Cherry Hill, $\mathrm{NJ}$ ) were dissolved in dimethylsulfoxide and added to cultures in $10 \mu \mathrm{l}$ volumes. Equivalent volumes of vehicle were added to parallel control cultures. In the experiments in which treatments with DGG, APV, $\mathrm{Co}^{2+}$, or GABA plus diazepam were combined with glutamate, glutamate was added to cultures $15 \mathrm{~min}$ after addition of the other agents.

Fura-2 analysis of intracellular calcium levels. In general, the procedure of Connor (1986) was used. Briefly, hippocampal cultures ( $3 \mathrm{~d}$ in culture) were loaded with fura-2/AM (Molecular Probes, Eugene, OR; $2 \mathrm{~mm}$ in DMSO diluted to a final incubation concentration of 3 $\mu \mathrm{M})$ for $30 \mathrm{~min}$, washed, and incubated for $60 \mathrm{~min}$ to allow hydrolysis of the ester. The cultures were viewed on a Zeiss ICM microscope with an RCA S.I.T. camera; the camera output was fed into a Quantex (Santa Clara, CA) QX7-210 Image Processing System, where it was converted to a $640 \times 480$ digital image (256 gray levels) and averaged for 500 msec ( 16 frames). The excitation wavelength was determined by a computer-controlled filter wheel that rotated between $350 \pm 10 \mathrm{~nm}$ and 380 $\pm 10 \mathrm{~nm}$ interference filters. Neutral density filters $(0.12 \mathrm{ND})$ were inserted in the excitation path to reduce bleaching of the fura- 2 and prevent saturation of the camera by the emission from the cell body. Because of the reduced excitation intensity, the fluorescent signal from the processes was close to background noise level; although measurable ratios were usually obtained from the processes, calcium concentrations were taken only from the soma. The fluorescent emission was filtered with a $495 \mathrm{~nm}$ long-pass emission filter. Fluorescent images were captured using each excitation filter; the ratio (R) of fluorescence intensity [(350 nm image $) /(380 \mathrm{~nm}$ image $)]$ was converted to calcium concentration using the formula $\mathrm{Ca}=\mathrm{K}_{\mathrm{d}}\left[\left(\mathrm{R}-\mathrm{R}_{\min }\right) /\left(\mathrm{R}_{\max }-\mathrm{R}\right)\right]\left(\mathrm{F}_{\mathrm{o}} / \mathrm{F}_{\mathrm{s}}\right)$ (Grynkiewicz et al., 1985). For our system, $R_{\min }=0.39 ; R_{\max }=11 ;\left(F_{0} / F_{S}\right)=$ $10 ; \mathrm{K}_{\mathrm{d}}=224$.

\section{Results}

\section{Generation of neuroarchitecture}

Cultures of hippocampal cells derived from 17-d-old rat embryos contained predominantly pyramidal-like neurons with distinct axonal and dendritic processes (Fig. 1; cf. Banker and Cowan, 1977, 1979; Mattson et al., 1988a). Previous studies used thymidine autoradiography to show that $10-20 \%$ of the cells have not yet undergone their last mitosis at the time of plating and can be observed to divide and differentiate as pyramidal-like neurons within the first 2 days in vitro (Banker and Cowan, 1977; Mattson et al., 1988a [Fig. 1]). Following mitosis in culture, mitotic sister neurons extend several short processes within $20 \mathrm{hr}$ (Fig. $1 A^{\prime}$ ). By $30 \mathrm{hr}$ the majority of neurons have differentiated an identifiable axon that elongates rapidly and several short dendrites that define the morphology of these neurons (Fig. 1 $A^{\prime \prime}$; cf. Dotti et al., 1988).

Since previous work in neuroblastoma cells indicated that mitotic sister cells tended to have similar morphologies (Solomon, 1981), we examined the possibility that mitotic relations might play a role in the generation of neuroarchitecture in normal mammalian CNS neurons. Two approaches were taken; one involved comparisons of neuronal morphologies by naive observers and the other involved quantitative analyses of morphology. Subjects $(n=7)$ were given 20 cards, each containing a tracing of one of a pair of sister neurons ( $3 \mathrm{~d}$ in culture), and were asked to pair neurons with similar shapes. On average, 33 $\pm 3 \%$ of the pairs were correctly matched as sister cells; this 

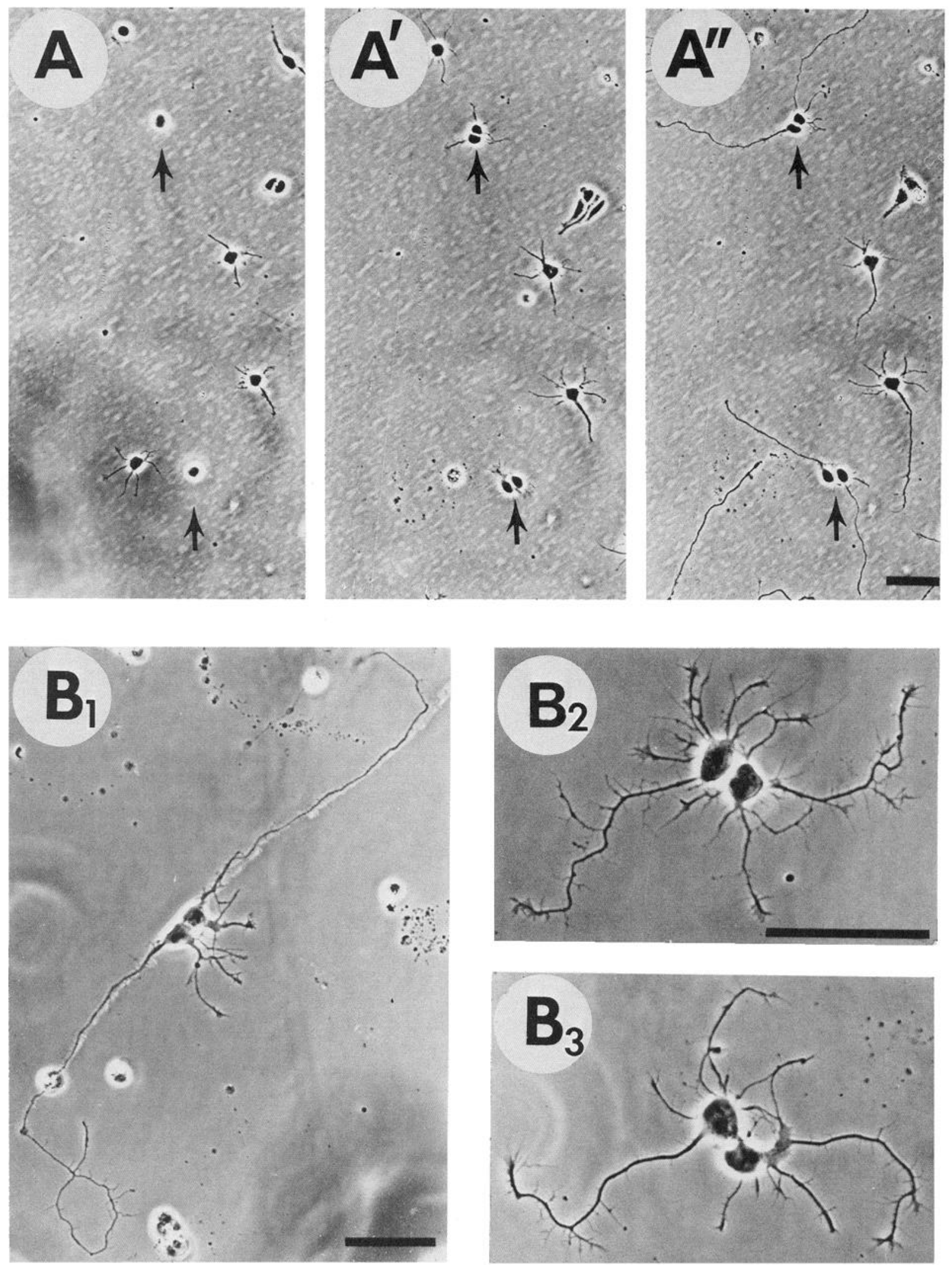

Figure 1. Morphological similarity of mitotic sister hippocampal pyramidal neurons. The upper 3 micrographs are of the same field of a culture dish at $6 \mathrm{hr}(A), 20 \mathrm{hr}(A)$, and $30 \mathrm{hr}\left(A^{\prime \prime}\right)$ after plating. $A$, Two single cells (arrows) have begun to divide by $6 \mathrm{hr}$ in culture as cleavage furrows are detectable. $A^{\prime}$, By $20 \mathrm{hr}$, cell division is complete and the sister neurons are beginning to elaborate neurites. $A^{\prime \prime}$, After $30 \mathrm{hr}$, the sister neurons have differentiated an axon (long process) and dendrites. The lower micrographs $\left(B_{1}-B_{3}\right)$ are representative examples of sister pyramidal neurons that had undergone mitosis between 4 and $20 \mathrm{hr}$ of culture; the micrographs were taken 3 days after plating. Scale bar, $50 \mu \mathrm{m}$. 
Sisters

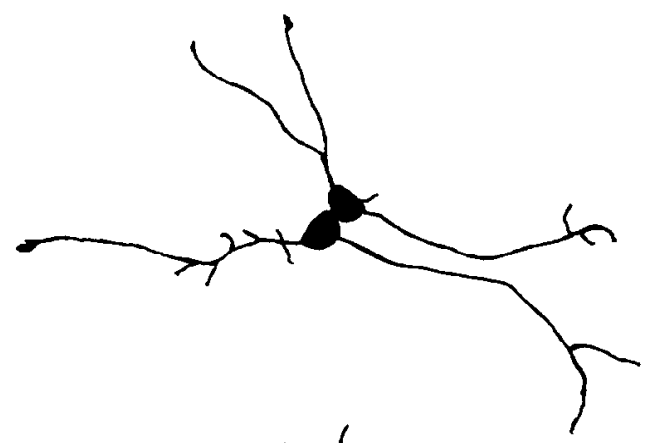

Figure 2. Comparison of morphologies of mitotic sister neuron pairs and non-sister neuron pairs. Tracings made on culture day 3 of sister neurons arising from a common progenitor (left) and non-sister neurons that, by chance, came to lie and grow in contact (right). Note that sister neurons have similar somal shapes and elaborate similar neuritic arbors. These tracings are representative of all 121 sister pairs and 24 nonsister pairs examined.
Non-Sisters

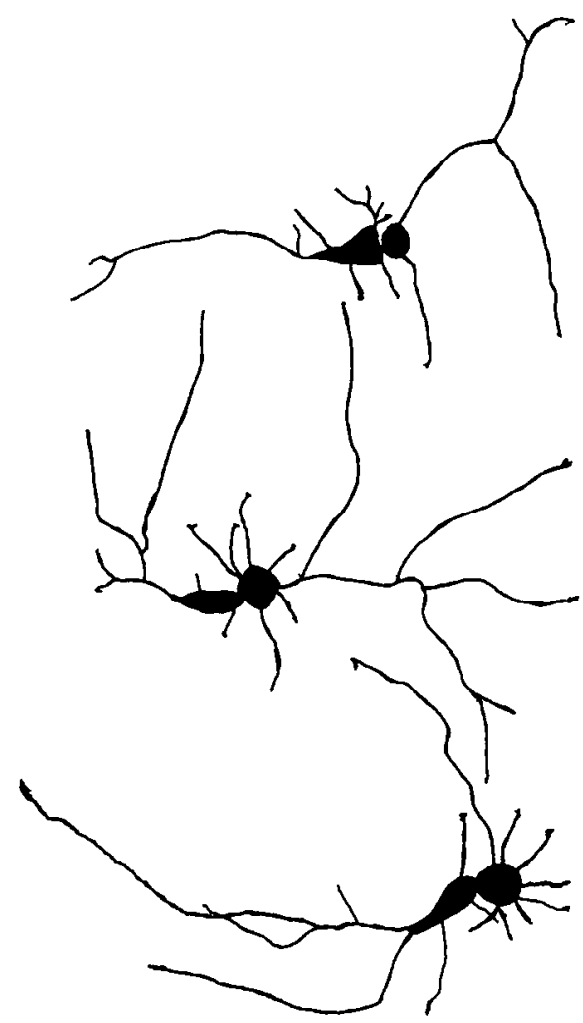

level of pairing was significantly greater than that obtained by chance $(2.9 \pm 1.8 \%$ of the pairs were correctly matched by blind pairing; $p<0.001$ ). The probability of matching 3 or more pairs by chance (out of 10 pairs) is 0.085 . Thus, there was a highly significant perceptual similarity in the morphologies of mitotic sister neurons. Examples of the similar morphologies of sister neurons are depicted in Figs. $1 B$ and 2 . Sister neurons generally had similar somal shapes and similarly shaped neuritic arbors (Figs. $1 B, 2$ ). In order to determine whether the local microenvironment or contact between sister neurons was influencing the generation of morphology, we examined unrelated (nonsister) pyramidal neurons that were in contact (see Materials and Methods). In non-sister neuron pairs the cell bodies of each neuron were often of clearly different shapes and neuritic arbors appeared dissimilar (Figs. 2 and $4, A, B$ ). Furthermore, naive observers matched non-sister cells at chance levels $-2.1 \pm 2.0 \%$ of the pairs were matched correctly-indicating that pairs of non-sister neurons did not generate similar shapes.

A more objective determination of morphological relatedness of mitotic sister neurons was made by measuring dendritic numbers and lengths (Table 1). There was a significant correlation between the number of primary dendrites of one neuron and the other of mitotic pairs $\left(\mathrm{R}^{2}=0.744, p<0.0001\right.$; Table 1$)$. Similarly, the length of dendritic arbors tended to be similar, with a significant correlation between sister neurons $\left(R^{\prime}=0.932\right.$, $p<0.0001$; Table 1). In non-sister neuron pairs there was no significant correlation between numbers of primary dendrites of each cell in the pair, nor was there a correlation in dendritic lengths (Table 1). Taken together, these results provide evidence that intrinsic neuronal properties related to mitotic history may be important in the generation of neuronal morphology in normal mammalian neurons.

\section{Glutamate-induced neurodegeneration}

The data above demonstrated that the generation of neuroarchitecture is similar in sister pyramidal neurons arising from a common progenitor. Since it is known that pyramidal neurons in different hippocampal regions are selectivcly vulncrable in several neurodegenerative disorders in which glutamate overactivity is implicated (Paul and Scheibel, 1986; VanHoesen et al., 1986; Maragos et al., 1987), we next asked whether neurons that are closely related in cell lineage also possess similar response systems for glutamate.

Cultures ( $5 \mathrm{~d}$ in culture) were exposed to $1 \mathrm{~mm}$ glutamate, and cell survival was assessed $4 \mathrm{hr}$ later. Glutamate killed 80$85 \%$ of the pyramidal neurons, leaving a glutamate-resistant subpopulation (Table 2). All sensitive neurons died within $4 \mathrm{hr}$ of glutamate exposure; resistant neurons survived through a 20 hr examination period. Similar percentages (15-20\%) of nonsister and sister pyramidal neurons were resistant to glutamate neurotoxicity (Table 2). Perhaps the most striking finding of the present study was that in all cases examined either both sisters died $(n=140)$ or both sisters lived $(n=26)$ when exposed to 1 mM glutamate (Fig. 3). When the time course of cell death in sister cell pairs was examined more closely (within the first 3 hr of glutamate exposure), it was observed that one cell in a pair would often die 30 to $60 \mathrm{~min}$ before the other cell. Selective vulnerability was conserved through at least 2 mitoses; in all cases examined, each member of 4 cells derived from the same 
Table 1. Comparisons of dendritic arbors in sister and non-sister cell pairs

\begin{tabular}{|c|c|c|c|}
\hline Parameter & Neuron A & $\begin{array}{l}\text { Sister } \\
\text { neuron B }\end{array}$ & $\begin{array}{l}\text { Non-sister } \\
\text { neuron B }\end{array}$ \\
\hline \multirow{5}{*}{$\begin{array}{l}\text { Number of primary } \\
\text { dendrites }\end{array}$} & 2 & $2.1 \pm 0.3^{a}$ & $4.5 \pm 0.7$ \\
\hline & 3 & $3.1 \pm 0.2^{a}$ & $3.8 \pm 0.6$ \\
\hline & 4 & $3.8 \pm 0.2^{a}$ & $4.0 \pm 1.0$ \\
\hline & 5 & $4.9 \pm 0.2^{a}$ & $3.4 \pm 0.7$ \\
\hline & 6 & $5.7 \pm 0.3^{a}$ & $3.5 \pm 0.6$ \\
\hline \multirow{6}{*}{$\begin{array}{l}\text { Length of dendritic } \\
\text { arbors }(\mu \mathrm{m})\end{array}$} & $75-100$ & $89.2 \pm 4.2^{a}$ & $113 \pm 31.4$ \\
\hline & $101-125$ & $114.2 \pm 2.7^{a}$ & $117 \pm 24.9$ \\
\hline & $126-150$ & $141.1 \pm 2.5^{a}$ & $110 \pm 22.7$ \\
\hline & $151-175$ & $160.2 \pm 4.9^{a}$ & $118 \pm 8.5$ \\
\hline & $176-200$ & $184.4 \pm 4.4$ & $105 \pm 23.3$ \\
\hline & $201-225$ & $204.6 \pm 8.0$ & - \\
\hline
\end{tabular}

Measurements were made on either sister neurons that arose from a final cell division and initiated outgrowth between 4 and $20 \mathrm{hr}$ after plating or on unrelated neurons that came to lie in contact with each other; measurements were made on culture day 3. For each cell pair, one cell (A) was used to establish a value for dendritic numbers and total dendritic length to which the other cell (B) was compared. Values represent the mean \pm SEM of determinations made on 5-11 sister cell pairs and 4-6 non-sister pairs. Correlation coefficients for sister cell pairs were highly significant for both dendrite numbers $\left(\mathrm{R}^{2}=0.744 ; p<0.0001\right)$ and dendrite lengths $\left(\mathrm{R}^{2}=0.932 ; p<0.0001\right)$.

${ }^{a} p<0.05-0.001$ compared to all other values.

precursor cell either died ( $n=9)$ or survived $(n=4)$ glutamate exposure. In order to rule out the possibility that the similar responses to glutamate in sister cells resulted from influences of local microenvironment or cell contact, we examined glutamate neurotoxicity in pairs of touching neurons that were not mitotic sisters. In 7 of the 24 cases examined, one neuron of the pair degenerated in response to glutamate while the other remained intact (e.g., Fig. 4), indicating that cell contact was probably not a determinant of whether neurons were sensitive or resistant to glutamate.

\section{Cellular mechanisms}

Previous studies demonstrated the potential for neurotransmitters, electrical activity, and calcium to regulate neuronal outgrowth and survival in several types of cultured neurons (Haydon et al., 1984; Cohan and Kater, 1986; Lankford et al., 1987; Mattson and Kater, 1987; Kater and Mattson, 1988; Kater et al., 1988; Lipton et al., 1988; Mattson, 1988; Mattson et al., 1988a, d). In the present study we examined the cellular basis of glutamate neurotoxicity in isolated non-sister pyramidal neurons and in mitotic pairs. Pharmacological manipulations were used to test which glutamate receptor types were involved in the neurotoxicity and whether the mechanism involved electrical activity and calcium influx. In addition, direct measurements of intracellular calcium were made using fura-2. In order to determine which receptor types were involved in glutamate toxicity, appropriate agonists and antagonists were employed (Watkins and Olverman, 1987). Kainate $(100 \mu \mathrm{M})$ and quisqualate $(100 \mu \mathrm{M})$ were each toxic to approximately $80 \%$ of mitotic pairs (Table 2); as with glutamate toxicity, either both cells in a pair died or both survived. NMDA (1 mM) did not cause cell death (Table 2). The general glutamate receptor antagonist DGG significantly reduced glutamate neurotoxicity in both individual isolated neurons and in mitotic pairs, whereas the NMDA receptor-specific antagonist APV did not alter glutamate toxicity (Table 2 ). We conclude that glutamate neurotox-
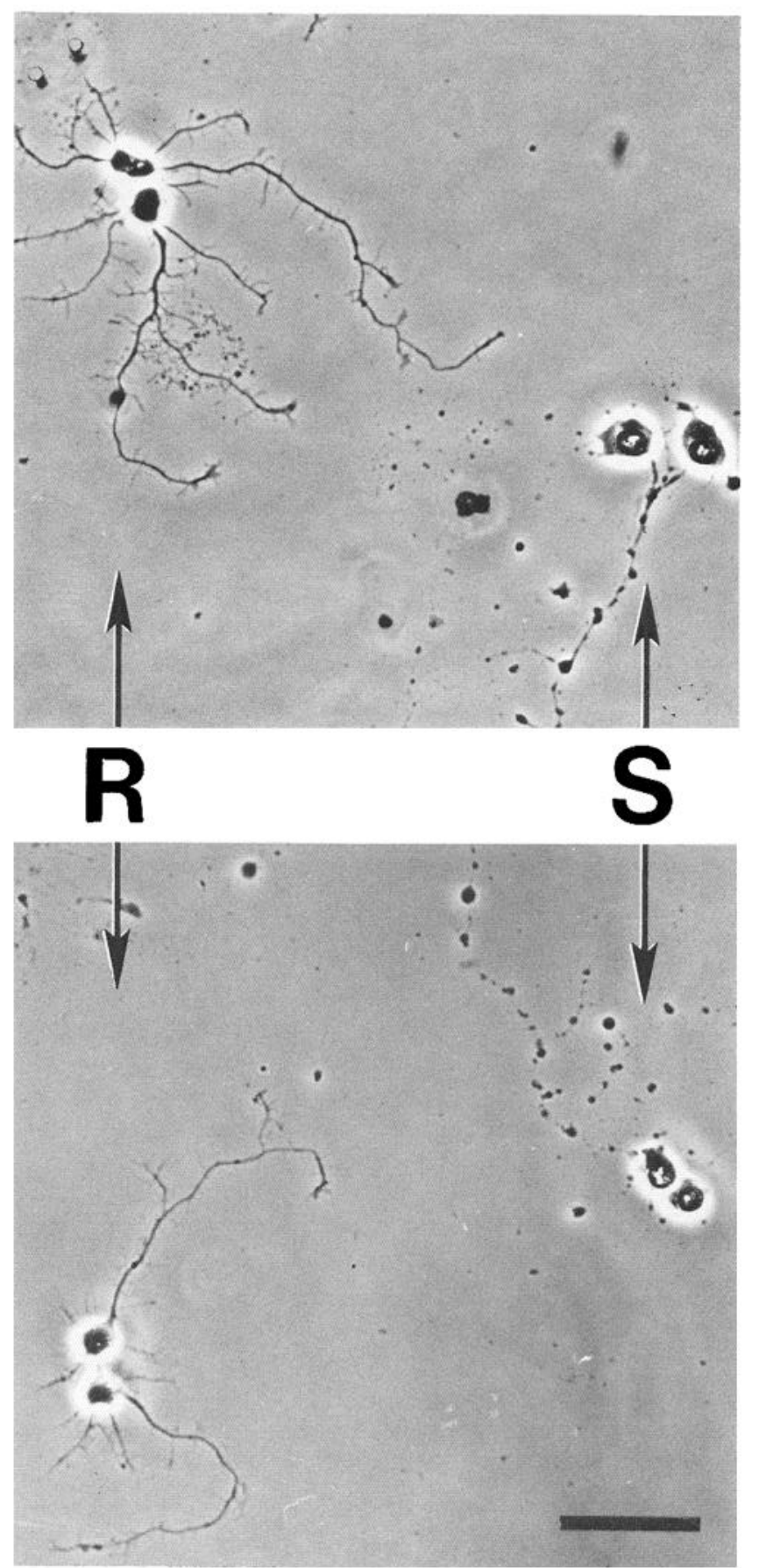

Figure 3. Sensitivity to glutamate neurotoxicity in mitotic sister neurons. The 2 micrographs are representative examples of sister pyramidal neurons $(n=166)$ that were either sensitive $(S)$ or resistant $(R)$ to glutamate neurotoxicity. Photographs were taken $4 \mathrm{hr}$ following exposure to $1 \mathrm{~mm}$ glutamate. Scale bar, $50 \mu \mathrm{m}$.

icity in all pyramidal neurons in the present study was mediated by glutamate receptors of the kainate/quisqualate types. In order to determine whether electrical activity was involved in glutamate neurotoxicity, we employed the inhibitory neurotransmitter GABA and its potentiator diazepam, a treatment that recently was found to reduce glutamate neurotoxicity in hippocampal cultures (Mattson and Kater, 1989). When cultures were pretreated with GABA and diazepam prior to glutamate 

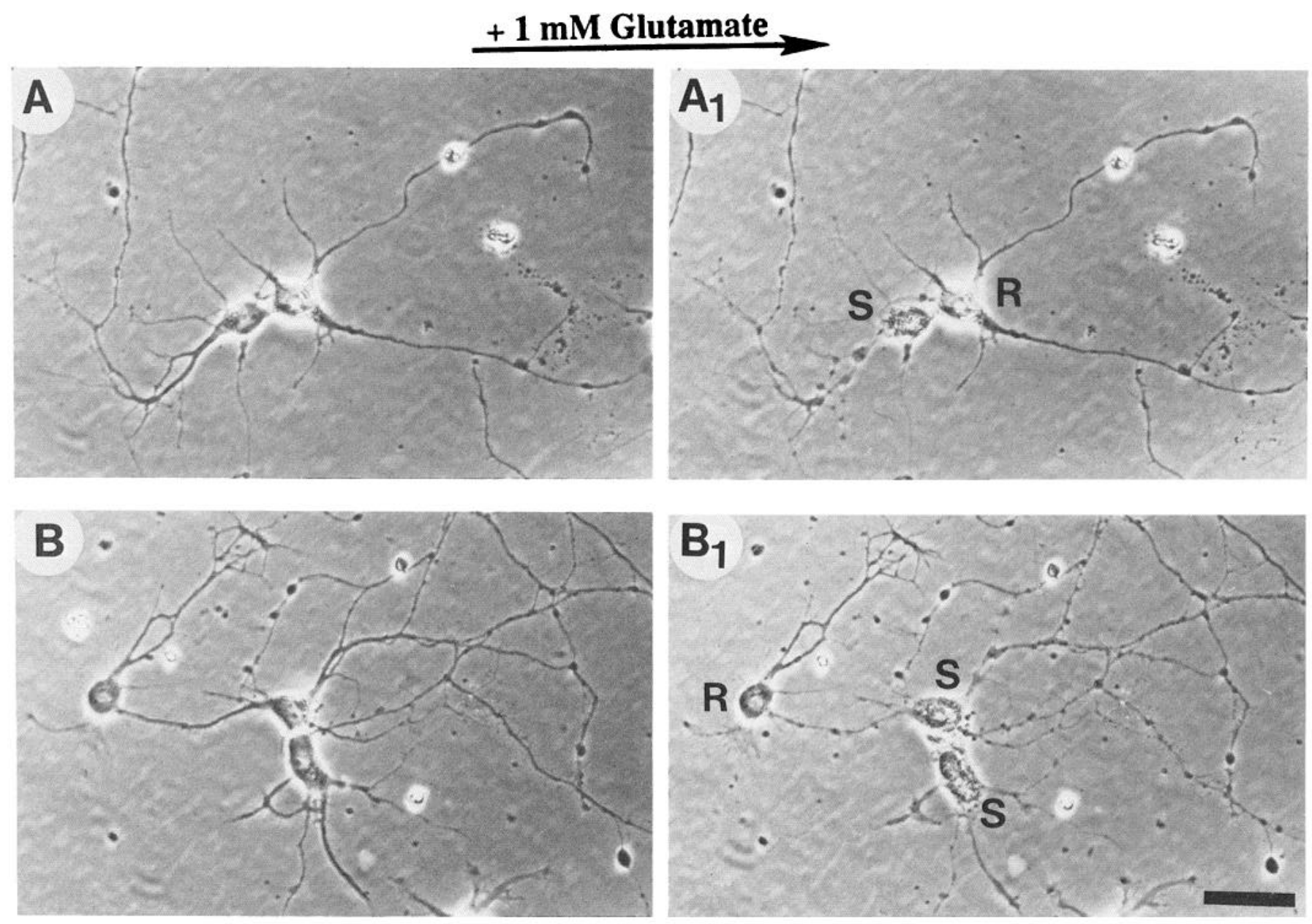

Figure 4. Non-sister neurons growing in contact differ in their morphologies and sensitivity to glutamate neurotoxicity. Phase-contrast micrographs of a non-sister neuron pair before treatment $(A$ and $B)$ and $4 \mathrm{hr}$ following exposure to $1 \mathrm{~mm}$ glutamate $\left(A_{l}\right.$, and $\left.B_{l}\right)$. Note that the paired cells have different somal shapes and elaborate different numbers of primary dendrites $(A, B)$. In $A$, it can be seen that one neuron of the pair was sensitive to glutamate neurotoxicity and degenerated $(S)$, while the other neuron was resistant $(R)$. In $B$, both cells in the pair were sensitive $(S)$, while another cell in the field was resistant $(R)$. Scale bar, $25 \mu \mathrm{m}$.

exposure, cell death was significantly reduced in both non-sister neurons and in mitotic pairs (Table 2). The mechanism of glutamate neurotoxicity apparently involved calcium influx since the calcium channel blocker $\mathrm{Co}^{2+}$ prevented glutamate neurotoxicity and calcium ionophore A23187 was neurotoxic (Table 2).

In order to directly test the effects of glutamate on intracellular calcium levels in mitotic sister neurons, the calcium-sensitive dye indicator fura-2 was used (Grynkiewicz et al., 1985; Connor, 1986; Cohan et al., 1987; Mattson et al., 1988d). Basal levels of intracellular calcium were similar in both glutamate-sensitive and -resistant neurons $(73 \pm 3.4 \mathrm{nM})$, and, interestingly, there was a significant correlation between rest levels of intracellular calcium within mitotic pairs (correlation coefficient, $0.621 ; p<$ 0.001 ). When cultures were exposed to $1 \mathrm{~mm}$ glutamate, we found that glutamate toxicity as assessed morphologically (Fig. 3 ) was always preceded by a dramatic rise in intracellular calcium levels in each neuron of a pair (Fig. 5). In glutamatesensitive neurons, intracellular calcium levels rose dramatically to $1000 \pm 260 \mathrm{~nm}(n=47)$, while in neurons resistant to glutamate $(n=9)$, calcium levels showed no significant rise (Fig. 5). The rises in intracellular calcium always preceded morpho- logical changes associated with toxicity by $30-60 \mathrm{~min}$. In mitotic pairs sensitive to glutamate, the large calcium rises were often observed to occur in one sister several min prior to a rise in the other cell. Thus, the changes in intracellular calcium levels observed with fura- 2 support the pharmacological data and point to glutamate-induced calcium influx through voltage-sensitive channels as the mechanism of glutamate neurotoxicity.

\section{Discussion}

The formation of neuronal circuitry requires that the component neurons elaborate neuritic arbors whose geometry and connections result in an ensemble appropriate for the function of that circuitry. The circuitry is, however, subject to modification. Modifications of neuroarchitecture may be adaptive as in learning and memory processes (Lee et al., 1980; Desmond and Levy, 1983; Chang and Greenough, 1984; Lynch, 1986) or disruptive as in neurodegenerative disorders (Mehraein et al., 1975; Buell and Coleman, 1979; Coyle et al., 1981; Paul and Scheibel, 1986; Maragos et al., 1987). General mechanisms operative in the development and modification of neuroarchitecture have been elusive but undoubtedly involve both genetic and environmental components. The results of the present study suggest that 
neuronal properties arising from mitotic history can be important both in the initial elaboration of neuritic architecture and in responses to environmental signals such as glutamate that modify neuroarchitecture.

The cellular basis for the similarity in shape of mitotic sister neurons is not known. However, our results indicate that the similar shapes of sister cells do not result from a similar microenvironment for outgrowth or from cell interactions mediated by contact. Indeed, non-sister cells that came to lie and grow in contact did not gencratc similar morphologies. Several explanations for the similarity of sister neuron shapes can be envisioned. Two reasonable possibilities are: (1) a genetic program for the generation of neuronal form that is determined earlier in development and stably inherited and expressed; or (2) a common cytoplasmic organization resulting from the position of the mitotic spindle, centrioles, or Golgi that is retained following mitosis and organizes neuritic outgrowth. Clearly, a great deal of further work is necessary to understand the cellular and molecular bases by which mitotic history determines cell morphology.

Recent data indicate that intracellular calcium levels may play an important role in the regulation of neurite outgrowth and synaptogenesis (Cohan et al., 1987; Mattson and Kater, 1987; Kater et al., 1988; Mattson et al., 1988a-c). Correlations have been established between the outgrowth status of neurites and intracellular calcium levels in both invertebrate (Cohan et al., 1987) and mammalian (Connor, 1986) systems. Furthermore, the available data suggest that quite specific aspects of the generation of neuritic arbors can be precisely controlled by intracellular calcium (Kater et al., 1988). For example, relatively modest reductions in calcium levels can inhibit growth cone motility and simultaneously promote neurite elongation (Mattson and Kater, 1987; Mattson et al., 1988e). Thus, calcium has the potential to determine even the more subtle details of neuronal morphology. In the present study we found that mitotic sister neurons tended to have similar basal levels of intracellular calcium as well as similar morphological characteristics. These findings are consistent with the possibility that neuronal properties arising from mitotic history may be important in determining both intracellular calcium levels and neuroarchitecture. Although it is inviting to consider that a causal relationship exists between intracellular calcium levels and specific neuronal shapes (or vice versa), no such correlations were observed in the present study. However, we recently found that different morphological classes of hippocampal neurons, including bipolar, stellate, dentate granule cells and pyramidal cells of Ammon's horn, possess different and characteristic intracellular calcium levels (Guthrie et al., 1988). Future experiments in which neuronal morphology is cxamined at higher levels of resolution and in which intracellular calcium levels are perturbed and the resulting effects on neuroarchitecture are examined should shed light on this problem.

We recently provided evidence that the mechanism of glutamate neurotoxicity in isolated hippocampal pyramidal neurons involves calcium influx through plasma membrane channels (Mattson et al., 1988a, d). Calcium is also believed to play a major role in glutamate neurotoxicity in other systems (Choi, 1987; Murphy et al., 1987). In the present study we found that calcium influx was a mediator of glutamate neurotoxicity in sister cells. Calcium influx preceded cell death, and the influx was both a necessary (glutamate-induced degeneration was prevented by $\mathrm{Co}^{2+}$ ) and a sufficient (A23187 caused cell death)
Table 2. Mechanism of glutamate neurotoxicity in isolated individual and mitotic sister hippocampal pyramidal neurons

\begin{tabular}{lcc} 
& \multicolumn{2}{l}{$\begin{array}{l}\text { Survival (\% of initial } \\
\text { number of neurons) }\end{array}$} \\
\cline { 2 - 3 } Treatment & $\begin{array}{l}\text { Non-sister } \\
\text { neurons }\end{array}$ & $\begin{array}{c}\text { Mitotic } \\
\text { sister neurons }\end{array}$ \\
\hline Control & $98 \pm 1.1$ & $98 \pm 1.3$ \\
Glutamate $(1 \mathrm{mM})$ & $15 \pm 2.3^{a}$ & $19 \pm 2.3^{a}$ \\
Kainate $(100 \mu \mathrm{M})$ & $18 \pm 4.1^{a}$ & $20 \pm 3.6^{a}$ \\
Quisqualate $(100 \mu \mathrm{M})$ & $23 \pm 3.9^{a}$ & $18 \pm 4.1^{a}$ \\
NMDA $(1 \mathrm{mM})$ & $99 \pm 1.0$ & $100 \pm 0$ \\
DGG $(2 \mathrm{mM})$ & $90 \pm 4.1$ & $92 \pm 1.5$ \\
DGG plus glutamate & $74 \pm 2.8^{b}$ & $74 \pm 2.7^{b}$ \\
APV $(1 \mathrm{mM})$ plus glutamate & $17 \pm 3.4^{a}$ & $20 \pm 3.5^{a}$ \\
Co ${ }^{2+}(100 \mu \mathrm{M})$ & $95 \pm 1.6$ & $97 \pm 1.6^{2+}$ \\
Co ${ }^{2+}$ plus glutamate & $73 \pm 2.9^{b}$ & $79 \pm 4.4^{b}$ \\
A23187 $(1 \mu \mathrm{M})$ & $1 \pm 0.7^{c}$ & $0 \pm 0.0^{c}$ \\
GABA $(10 \mu \mathrm{m})$-diazepam $(1 \mu \mathrm{M})$ & & \\
$\quad$ plus glutamate & $83 \pm 2.1^{b}$ & $87 \pm 1.9^{b}$ \\
\hline
\end{tabular}

5 -d-old cultures were exposed to the indicated treatments and cell viability was assessed $4 \mathrm{hr}$ later. Values represent the mean \pm SEM of determinations made on neurons in 3 separate cultures; 4 fields/culture were examined and each field contained approximately 50 isolated pyramidal neurons and 3-8 mitotic pairs.

" $p<0.001$ compared to control.

${ }^{s} p<0.05-0.01$ compared to control; $p<0.001$ compared to the glutamate value. $p<0.001$ compared to control and to the glutamate value.

stimulus to cause neurodegeneration. The receptors mediating the glutamate responses in mitotically related neurons were of the kainate/quisqualate type since these agonists (but not NMDA) mimicked glutamate's actions, while the general glutamate receptor antagonist DGG prevented glutamate neurotoxicity and the NMDA-specific antagonist APV was ineffective. Activation of the glutamate receptors led to calcium influx, apparently through voltage-dependent plasma membrane channels, since GABA plus diazepam prevented the effects of glutamate. Indeed, Connor et al. (1988) have recently shown that GABA reduces glutamate-induced calcium influx in mature hippocampal CAl pyramidal neurons, and we recently found that GABA plus diazepam prevents glutamate-induced degeneration in isolated hippocampal neurons (Mattson and Kater, 1989). The best explanation for these results is that glutamate-resistant neurons lack glutamate receptors linked to calcium influx and cell death. The latter possibility is consistent with results obtained in situ that documented differences in glutamate receptors between hippocampal pyramidal neurons using electrophysiological techniques (Collinridge et al., 1983a, b; Dingledine, 1983). Furthermore, based on studies of excitatory amino acid neurotoxicity in mouse cortical cultures, Koh et al. (1986) postulated that glutamate-resistant neurons possess a different set of glutamate receptors which confer resistance to glutamate. The results of the present study suggest that the selective vulnerability of different pyramidal neurons may result from intrinsic differences in the expression of glutamate receptors linked to calcium influx.

While our data suggest that cell lineage differences may lead to differences in neuroarchitecture and response systems for neurotransmitters, it is important to also consider that ongoing communications between closely related neurons might contribute to their similar morphologies and responses to environmental signals such as glutamate. For example, if mitotic pairs 

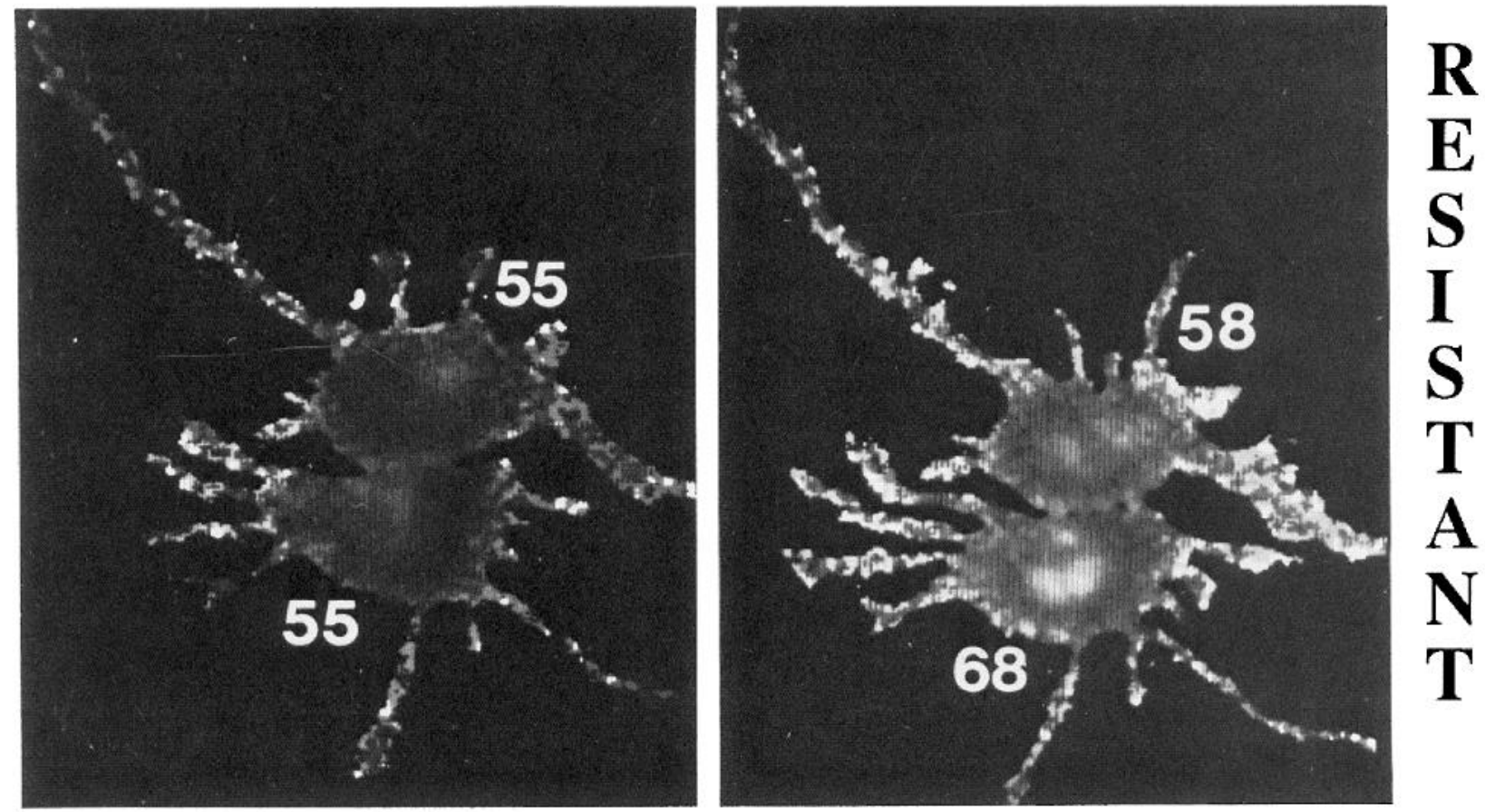

\section{Glutamate}
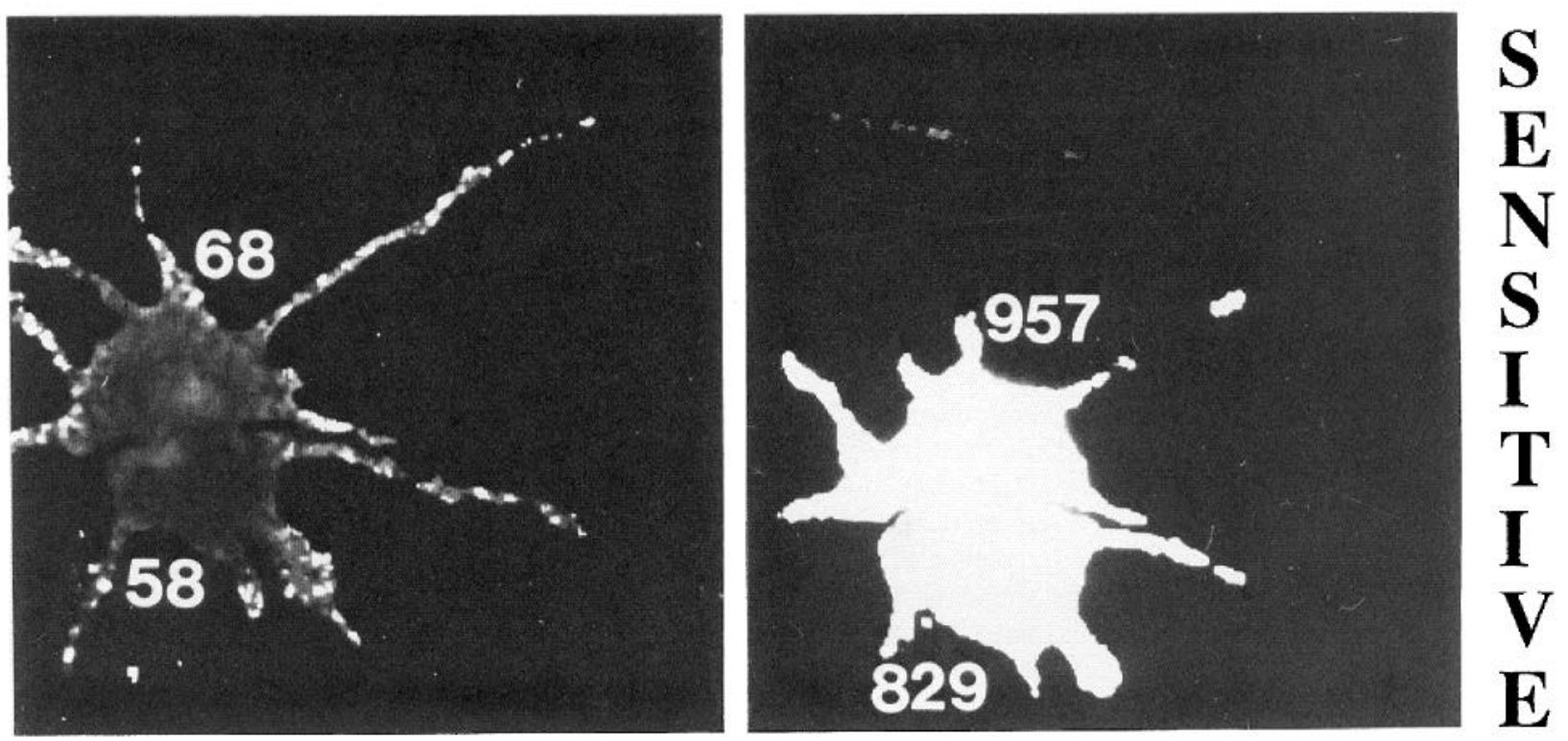

Figure 5. Glutamate neurotoxicity is linked to a rise in levels of free intracellular calcium. The fluorescent calcium indicator dye fura- 2 was used to monitor levels of free intracellular calcium. Two pyramidal sister cell pairs are shown prior to exposure to $1 \mathrm{~mm}$ glutamate $(l e f t)$ and $60 \mathrm{~min}$ following exposure to glutamate (right). The upper sister cell pair was resistant to the effects of glutamate and showed essentially no change in calcium levels (numbers adjacent to cells are the intracellular calcium concentrations of each cell in nM). In contrast, the lower cell pair was sensitive to glutamate and showed a dramatic rise in calcium levels. In all 56 cases examined, sister cells either showed no change in intracellular calcium levels and survived exposure to glutamate or showed a large calcium response and subsequently degenerated (cf. Fig. 3). In sensitive neurons, the onset of the calcium rise preceded the appearance of morphological injury.

were electrically coupled (O'Beirne et al., 1987), then only one neuron of a mitotic pair might be sensitive to glutamate and communicate outgrowth or excitotoxic signals to the other neuron. However, several lines of evidence point to the mitotic relation of the neurons, and not their physical proximity, as being of major importance. Thus, when individual neurons came to lie and grow in physical contact, these neurons did not elab- orate similar morphologies and often showed different responses to glutamate. In addition, if a signal was communicated from one neuron to the other, then it would be expected that the percentage of sister neurons sensitive to glutamate toxicity would be greater than the number of vulnerable individual isolated neurons; this was not the case (Table 2). Furthermore, rises in intracellular calcium and subsequent cell death often occurred 
in one neuron of a mitotic pair prior to occurrence in the other neuron; this time delay would not be expected if electrical coupling was mechanistically involved. Since hippocampal neurons that arise from common progenitors are likely to occupy similar regions within Ammon's horn, their lineage probably contributes to their particular neuroarchitectural properties and sensitivities to glutamate, a possibility supported by our data.

It is now generally believed that a great deal of structural modification "adjustment" of neuronal circuits occurs both during development and in adult life (Purves and Hadley, 1985). Clearly, 2 major mechanisms in the formation and modification of neuronal circuitry involve changing neuronal number and neuronal geometry. For example, synaptic rearrangements and cell death appear to play important roles in the formation of functional neuromuscular systems (Oppenheim, 1987). The results of the present study demonstrate that the mitotic history of hippocampal neurons can determine whether they live or die, as well as their morphological features. These results suggest that the mitotic history of a neuron can influence both its presence and its specific connectivity within neuronal circuits. During hippocampal development pyramidal neurons may utilize intrinsic cues to generate their morphology and to respond to external signals such as glutamate. For example, natural cell death or dendritic pruning may occur during development in order to adjust neuronal circuitry to a proper functional state. The expression of response systems to exogenous signals such as glutamate may determine which neurons are pruned and which are spared and grow. Recent studies of cerebellar development have demonstrated that Purkinje cells descend from a small number of progenitor cells selected to be the sole source of the adult population (Herrup, 1986). If similar mechanisms are operative during hippocampal development, then it is possible that different groups of neurons arising from particular progenitors might exhibit differences in cell morphology and in their outgrowth and survival responses to environmental signals such as the neurotransmitter glutamate. Such genetically determined neuronal properties may influence both the expression of neuronal form and the modification of this form by environmental factors.

Finally, the present findings are consistent with a possible role for cell lineage relationships in the selective vulnerability of particular groups of hippocampal pyramidal neurons. Pyramidal neurons in regions $\mathrm{CA} 1$ and $\mathrm{CA} 3$ are selectively lost in Alzheimer's diesease (Mehraein et al., 1975; VanHoesen et al., 1986), epilepsy (Scheibel et al., 1974; Paul and Scheibel, 1986), and stroke (Schwarcz et al., 1984). Glutamate overactivity has been implicated in the hippocampal neurodegeneration associated with each of these disorders (Schwarcz et al., 1984; Maragos et al., 1987; Mattson, 1988). The present data demonstrate that a subpopulation of pyramidal neurons is sensitive to glutamate-induced neurodegeneration and that this selective vulnerability is related to mitotic history. It is not known whether pyramidal neurons within the different hippocampal regions arise through common cell lineages, but the present data are consistent with this possibility.

\section{References}

Banker, G. A., and W. M. Cowan (1977) Rat hippocampal neurons in dispersed cell culture. Brain Res. 126: 397-425.

Banker, G. A., and W. M. Cowan (1979) Further observations on hippocampal neurons in dispersed cell culture. J. Comp. Neurol. 187: $469-494$.
Buell, S. J., and P. D. Coleman (1979) Dendritic growth in the aged human brain and failure of growth in senile dementia. Science 206 : 854-856.

Chang, F. L. F., and W. T. Greenough (1984) Transient and enduring morphological correlates of synaptic activity and efficacy change in the rat hippocampal slice. Brain Res. 309: 35-46.

Choi, D. W. (1987) Ionic dependence of glutamate neurotoxicity. J. Neurosci. 7: 369-379.

Cohan, C. S., and S. B. Kater (1986) Suppression of neurite elongation and growth cone dynamics by electrical activity. Science 232: 16381640 .

Cohan, C. S., J. A. Connor, and S. B. Kater (1987) Electrically and chemically mediated increases in intracellular calcium in neuronal growth cones. J. Neurosci. 7: 3588-3599.

Collinridge, G. I., S. J. Kehl, and H. McLennan (1983a) The antagonism of amino acid-induced excitations of rat hippocampal $\mathrm{CA}_{1}$ neurons in vitro. J. Physiol. (Lond.) 334: 19-31.

Collinridge, G. I., S. J. Kehl, and H. McLennan (1983b) Excitatory amino acids in synaptic transmission in the Schaffer collateral-commissural pathway of the rat hippocampus. J. Physiol. (Lond.) 334: 34-46.

Connor, J. A. (1986) Digital imaging of free calcium changes and of spatial gradients in growing processes in single mammalian central nervous system cells. Proc. Natl. Acad. Sci. USA 83: 6179-6183.

Connor, J. A., W. J. Wadman, P. E. Hockberger, and R. K. S. Wong (1988) Sustained dendritic gradients of $\mathrm{Ca}^{2+}$ induced by excitatory amino acids in CAl hippocampal neurons. Science 240:649-653.

Coyle, J. T., S. J. Bird, R. H. Evans, R. L. Gulley, J. V. Nadler, W. J. Nicklas, and J. W. Olney (1981) Excitatory amino acid neurotoxins: Selectivity, specificity, and mechanism of action. Neurosci. Res. Prog. Bull. 19: 331-427.

Desmond, N., and W. B. Levy (1983) Synaptic correlates of associative potentiation/depression: An ultrastructural study in the hippocampus. Brain Res. 265: 21-31.

Dingledine, R. (1983) N-methylaspartate activates voltage-dependent calcium conductance in rat hippocampal pyramidal cells. J. Physiol. (Lond.) 343: 385-405.

Dotti, C. G., C. A. Sullivan, and G. A. Banker (1988) The establishment of polarity by hippocampal neurons in culture. J. Neurosci. 8 : 1454-1468.

Fonnum, F. (1984) Glutamate: A neurotransmitter in mammalian brain. J. Neurochem. 42: 1-11.

Goodman, D. S. (1982) In Neuronal Development, N. C. Spitzer, ed., pp. 171-212, Plenum, New York.

Greene, L. A., and E. M. Shooter (1980) The nerve growth factor. Annu. Rev. Neurosci. 4: 353-402.

Grynkiewicz, G., M. Poenie, and R. Y. Tsien (1985) A new generation of calcium indicators with greatly improved fluorescence properties. J. Biol. Chem. 260: 1440-1447.

Guthrie, P. B., M. P. Mattson, L. Mills, and S. B. Kater (1988) Calcium homeostasis in molluscan and mammalian neurons: Neuron-selective set-point of calcium rest concentration. Soc. Neurosci. Abstr. 14: 582.

Haydon, P. G., D. P. McCobb, and S. B. Kater (1984) Serotonin selectively inhibits growth cone dynamics and synaptogenesis of specific identified neurons of Helisoma. Science 226: 561-564.

Herrup, K. (1986) Cell lineage relationships in the development of the mammalian CNS: Role of cell lineage in control of cerebellar Purkinje cell number. Dev. Biol. 115: 148-158.

Herrup, K. (1987) Roles of cell lineage in the developing mammalian brain. Curr. Top. Dev. Biol. 21: 65-97.

Kater, S. B., and M. P. Mattson (1988) Extrinsic and intrinsic regulators of neurite outgrowth and synaptogenesis in isolated, identified Helisoma neurons in culture. In Cell Culture Approaches to Invertebrate Neuroscience, D. J. Beadle and S. B. Kater, eds., pp. 1-32. Academic, London.

Kater, S. B., M. P. Mattson, C. S. Cohan, and J. A. Connor (1988) Calcium regulation of the neuronal growth cone. Trends Neurosci. 11: 315-321.

Koh, J., S. Peters, and D. W. Choi (1986) Neurons containing NADPHdiaphorase are selectively resistant to quinolinate toxicity. Science 234: 73-76.

Lankford, K. L., F. G. DeMello, and W. L. Klein (1987) A transient embryonic dopamine receptor inhibits growth cone motility and neurite outgrowth in a subset of avian retina neurons. Neurosci. Lett. 75: 169-174. 
Lasek, R. J., and M. M. Black (1988) Intrinsic Determinants of Neuronal Form and Function, Liss, New York.

Lctourncau, P. C. (1985) Axonal growth and guidance. In Molecular Bases of Neuronal Development, G. M. Edelman, W. E. Gall, and M. Cowan, eds., pp. 269-294, Wiley, New York.

Levi-Montalcini, R., and P. U. Angeletti (1968) Nerve growth factor. Physiol. Rev. 48: 534-569.

Lipton, S. A., M. P. Frosch, M. D. Phillips, D. L. Tauck, and E. Aizenman (1988) Nicotinic antagonists enhance process outgrowth by rat retinal ganglion cells in culture. Science 239: 1293-1296.

Luskin, M. B., A. L. Pearlman, and J. R. Sanes (1987) Cell lineage in mouse cerebral cortex studied with a retroviral marker. Soc. Neurosci. Abstr. 13: 183.

Lynch, G. (1986) Synapses, Circuits, and the Beginnings of Memory, MIT Press, Cambridge, MA.

Maragos, W. F., J. T. Greenamyre, J. B. Penny, and A. B. Young (1987) Glutamatc dysfunction in Alzheimer's disease: An hypothesis. Trends Neurosci. 10: 65-68.

Mattson, M. P. (1988) Neurotransmitters in the regulation of neuronal cytoarchitecture. Brain Res. Rev. 13: 179-212.

Mattson, M. P., and S. B. Kater (1987) Calcium regulation of neurite elongation and growth cone motility. J. Neurosci. 7: 4034-4043.

Mattson, M. P., and S. B. Kater (1988a) Fibronectin-like immunoreactivity in Helisoma buccal ganglia: Evidence that an endogenous fibronectin-like molecule promotes neurite outgrowth. J. Neurobiol. 19: 233-250.

Mattson, M. P., and S. B. Kater (1988b) Isolated hippocampal neurons in cryopreserved long-term cultures: Development of neuroarchitecture and sensitivity to NMDA. Int. J. Dev. Neurosci. 6: 439-452.

Mattson, M. P., and S. B. Kater (1989) Excitatory and inhibitory neurotransmitters in the generation and degeneration of hippocampal neuroarchitecture. Brain Res. 478: 337-348.

Mattson, M. P., P. Dou, and S. B. Kater (1988a) Outgrowth-regulating actions of glutamate in isolated hippocampal pyramidal neurons. $\mathrm{J}$. Neurosci. 8: 2087-2100.

Mattson, M. P., R. E. Lee, M. E. Adams, P. B. Guthrie, and S. B. Kater (1988b) Interactions between entorhinal axons and target hippocampal neurons: A role for glutamate in the development of hippocampal circuitry. Neuron $1: 865-876$.

Mattson, M. P., A. Taylor-Hunter, and S. B. Kater (1988c) Neurite outgrowth in individual neurons of a neuronal population is differentially regulated by calcium and cyclic AMP. J. Neurosci. 8: 17041711.

Mattson, M. P., P. B. Guthrie, and S. B. Kater (1988d) Intracellular messengers in the generation and degeneration of hippocampal neuroarchitecture. J. Neurosci. Res. 21: 447-464.

Mattson, M. P., P. B. Guthrie, and S. B. Kater (1988e) Components of neurite outgrowth which determine neuronal cytoarchitecture: Influence of calcium and the growth substrate. J. Neurosci. Res. 20: 331-345.

Mehraein, P., M. Yamada, and E. Tarnowska-Dziduszko (1975) Quantitative study on dendrites and dendritic spines in Alzheimer's disease and senile dementia. Adv. Neurol. 12: 453-458.

Meldrum, B. (1985) Possible therapeutic applications of antagonists of excitatory amino acid neurotransmitters. Clin. Sci. 68: 113-132.

Murphy, S. N., S. A. Thayer, and R. J. Miller (1987) The effects of excitatory amino acids on intracellular calcium in single mouse striatal neurons in vitro. J. Neurosci. 7: 4145-4158.

O'Beirne, M., A. G. M. Bulloch, and B. A. MacVicar (1987) Dye and electronic coupling between cultured hippocampal neurons. Neurosci. Lett. 78: 265-269.

Oppenheim, R. W. (1987) Muscle activity and motor neuron death in the spinal cord of the chick embryo. In Setective Neuronal Death, G. Bock and M. O'Connor, eds., pp. 96-107, Wiley, New York.

Paul, L. A., and A. B. Scheibel (1986) Structural substrates of epilepsy. Adv. Neurol. 44: 775-786.

Purves, D., and R. D. Hadlcy (1985) Changes in the dendritic branching of adult mammalian neurons. Nature 315: 404-406.

Rothman, S. (1984) Synaptic release of excitatory amino acid neurotransmitter mediates anoxic neuronal death. J. Neurosci. 4: 18841891.

Scheibel, M. E., P. H. Crandall, and A. B. Scheibel (1974) The hippocampal-dentate complex in temporal lobe epilepsy. Epilepsia 15: $55-80$.

Schwarcz, R., A. C. Foster, E. D. French, W. O. Whetsell, and C. Kohler (1984) Excitotoxic models for neurodegenerative disorders. Life Sci. 35: $19-32$.

Sloviter, R. S. (1983) "Epileptic" brain damage in rats induced by sustained electrical stimulation of the perforant path. I. Acute electrophysiological and light microscopic studies. Brain Res. Bull. 10: 675-697.

Solomon, F. (1981) Specification of cell morphology by endogenous determinants. J. Cell Biol. 90: 547-553.

Stent, G. S., D. A. Weisblat, S. S. Blair, and S. L. Zackson (1982) In Neuronal Development, N. C. Spitzer, ed., pp. 1-44, Plenum, New York.

Sulston, J. E., E. Schierenberg, J. G. White, and J. N. Thompson (1983) The embryonic cell lineage of the nematode Caenorhabditis elegans. Dev. Biol. 100: 64-119.

VanHoesen, G. W., B. T. Hyman, and A. R. Damasio (1986) Cellspecific pathology in neural systems of the temporal lobe in Alzheimer's disease. Prog. Brain Res. 70: 321-335.

Watkins, J. C., and H. J. Olverman (1987) Agonists and antagonists for excitatory amino acid receptors. Trends Neurosci. 10: 265-272. 